\title{
Blue-Light Therapy for Acne Vulgaris: A Systematic Review and Meta-Analysis
}

\author{
Anna Mae Scott, $P b D^{1}$ \\ Paulina Steblik, $P b D^{1}$ \\ Justin Clark, BA ${ }^{1}$ \\ Dexing Zhang, $P b D^{2}$ \\ Zuyao Yang, $P b D^{3}$ \\ Tammy Hoffmann, $P b D^{1}$ \\ Chris Del Mar, $M D^{1}$ \\ Paul Glasziou, PbD ${ }^{1}$
}

${ }^{1}$ Centre for Research in Evidence-Based Practice, Bond University, Robina,

Queensland, Australia

${ }^{2}$ Division of Family Medicine and Primary Health Care, Jockey Club School of Public Health and Primary Care, The Chinese University of Hong Kong, Shatin, Hong Kong

${ }^{3}$ Division of Epidemiology, Jockey Club School of Public Health and Primary Care, The Chinese University of Hong Kong, Shatin, Hong Kong

Conflicts of interest: No funding or other material support was sought or received to perform this work specifically, bowever, several of the authors (T.H., C.D.M., P.G.) are recipients of funding from the National Health and Medical Research Council Centre for Research Excellence in Minimising Antimicrobial Resistance from Acute Respiratory Infections (CREMARA), within whose remit the present review was conducted 2 of the authors' salaries (A.M.S., J.C.) are partially funded by CREMARA

\section{CORRESPONDING AUTHOR}

Anna Mae Scott, PhD

Centre for Research in Evidence-Based

Practice, Bond University

14 University Drive

Robina, QLD 4226, Australia

ascott@bond.edu.au

\begin{abstract}
PURPOSE Antibiotic use in acne treatment raises concerns about increased resistance, necessitating alternatives. We assessed the effectiveness of blue-light therapy for acne.
\end{abstract}

METHODS We analyzed randomized controlled trials comparing blue light with nonlight interventions. Studies included people of any age, sex, and acne severity, in any setting, and reported on investigator-assessed change in acne severity, patients' assessment of improvement, change in inflammatory or noninflammatory lesions, and adverse events. Where data were sufficient, mean differences were calculated.

RESULTS Eighteen references (14 trials) including 698 participants were included. Most of the trials were small and short ( $<12$ weeks) and had high risk of bias. Investigator-assessed improvement was quantitatively reported in 5 trials, of which 3 reported significantly greater improvement in blue light than comparator, and 2 reported improvement. Patients' assessments of improvement were quantitatively reported by 2 trials, favoring blue light. Mean difference in the mean number of noninflammatory lesions was nonsignificant between groups at weeks 4,8 , and 10-12 and overall (mean difference $[\mathrm{MD}]=3.47 ; 95 \% \mathrm{Cl},-0.76$ to $7.71 ; P=0.11$ ). Mean difference in the mean number of inflammatory lesions was likewise nonsignificant between groups at any of the time points and overall $(\mathrm{MD}=0.16 ; 95 \% \mathrm{Cl},-0.99$ to $1.31 ; P=0.78)$. Adverse events were generally mild and favored blue light or did not significantly differ between groups.

CONCLUSION Methodological and reporting limitations of existing evidence limit conclusions about the effectiveness of blue light for acne. Clinicians and patients should therefore consider the balance between its benefits and adverse events, as well as costs.

Ann Fam Med 2019;17:545-543. https://doi.org/10.1370/afm.2445.

\section{INTRODUCTION}

A pproximately 50 million Americans have acne vulgaris ${ }^{1}$ it is the eighth most prevalent disease globally ${ }^{2}$ and one of the most common reasons for clinical consultations ${ }^{3}$ including among primary care physicians and general practitioners (GPs). Among respondents to the James Lind Alliance's Acne Priority Setting Partnership survey, for example, $65 \%$ reported having sought help for acne from their GP or family doctor (higher than from a dermatologist, $45 \%$; a pharmacist, $34 \%$; or any other source).$^{4}$

Several effective pharmacological treatments for acne exist, such as topical benzyl peroxide and topical or oral retinoids and antibiotics. With antibiotics, however, there is increasing concern about the global development of resistance from the use of topical and oral antibiotics. The incidence of resistance in Proprionibacterium acnes has been increasing: more than $50 \%$ of $P$ acnes strains are resistant to erythromycin in Egypt, France, Greece, Italy, Spain, and the United Kingdom and to clindamycin in Egypt, Greece, Hong Kong, Italy, and Spain. ${ }^{5,6}$ This resistance not only reduces the effectiveness of antibiotic treatment for acne but also can 
spread to untreated contacts and hence affect greater population antibiotic resistance patterns.

Alternatives to antibiotics for acne treatment are thus desirable. One recent option is the use of various forms of light therapy, particularly blue-light therapy. Light in the 407- to 420-nm wavelength range has been shown to have a bactericidal effect on $P$ acnes. ${ }^{7}$ The proposed mechanism is the excitation of bacterial porphyrins (coproporphyrin III and protoporphyrin IX) leading to the release of singlet oxygen and reactive free radicals that exert bactericidal effects. ${ }^{3,8}$

The US Food and Drug Administration (FDA) has approved light-emitting diode (LED) devices (blue, red, and blue/red light devices) for at-home use, ${ }^{9}$ and the market for light therapy devices for acne is growing: the first device received FDA approval in 2009, and 35 devices are on the market now. ${ }^{10}$ Patient interest in these devices is correspondingly increasing, with "Which physical therapies, including lasers and other light-based treatments, are safe and effective in treating acne?" being the top acne treatment uncertainty identified by patients responding to the James Lind Alliance survey, at 35\% of respondents. ${ }^{4}$ Given the increased availability over-the-counter treatments, patient interest, and decreasing price-as well as the concerns about adverse events and antibiotic resistance caused by present treatments-interest is likely to keep increasing.

Application of blue light does require considerable commitment by the user, however, with typical at-home devices being used twice a day for 30 to 60 minutes for 4 to 5 weeks. Therefore, evidence of the size and sustainability of any effect would be important to potential users. A 2016 Cochrane review of all types of light therapy for acne found 71 trials, with little evidence for most, but low certainty of evidence for green or blue lights. ${ }^{11}$ Further trials of blue light have been published since, however. We therefore undertook a systematic review of randomized controlled trials examining the effects of blue-light therapy for acne compared with any other nonlight therapy, in people of any age, sex, and acne severity. The present systematic review will also underpin a proposed entry for blue-light therapies for acne in the Handbook of Non-Drug Interventions (HANDI), which aims to promote effective nondrug treatments for general practice or primary care. ${ }^{12}$

\section{METHODS}

\section{Protocol}

The protocol for this systematic review was registered on PROSPERO: http://www.crd.york.ac.uk/PROSPERO/ display_record.php?ID=CRD42018090053.

\section{Included Studies}

We included randomized controlled trials of any design (eg, parallel, crossover), in which randomization was by individual or by body part (eg, split face studies); participants of any age, sex, and severity of acne were included. Included studies investigated light treatment of restricted wavelength, in which blue light was a major component. The comparator could be any intervention other than light (including placebo; topical agents such as retinoids, benzoyl peroxide, or antibiotics; or oral antibiotics or isotretinoin). Nonrandomized study designs were excluded.

The primary outcome was investigator-assessed change in acne severity. Secondary outcomes included patient's global assessment of improvement, changes in inflammatory lesions (papules, pustules, nodules), changes in noninflammatory lesions (open and closed comedones), and adverse events.

\section{Search Strategy}

We searched PubMed, the Cochrane Central Register of Controlled Trials (via Wiley), Embase (via Elsevier), CINAHL (via EBSCO), and the Web of Science Core Collection (via Clarivate Analytics) from inception to March 5, 2018; we searched the clinical trial registries (clinicaltrials.gov and the World Health Organization's who.int) on August 7, 2018 (Supplemental Appendix, available at http://www.AnnFamMed.org/ content/17/6/545/suppl/DC1/). We also did forward and backward citation searches of the included studies (on August 7, 2018). No date or language restrictions were applied.

\section{Screening and Data Extraction}

Screening of the literature was conducted by 2 authors independently, first by title/abstract and subsequently in full text. Discrepancies were resolved by consensus or referring to a third author if necessary. Three data extraction forms were predesigned and piloted for this review: Table of Characteristics form, Primary and Secondary Outcomes data form, and Risk of Bias form. Data from included studies were extracted independently in pairs into the data extraction forms. Discrepancies were resolved by discussion or by reference to a third author.

\section{Risk of Bias}

The risk of bias was assessed with the Cochrane Collaboration's Risk of Bias tool, ${ }^{13}$ by 2 authors independently, with discrepancies resolved by discussion.

\section{Statistical Analysis}

A meta-analysis was conducted where data were sufficient to pool. The results are presented as mean differences with $95 \%$ CIs via a random effects model; there 
were sufficient data to present outcomes at weeks 4 , 8 , and 10-12. Because of the general paucity of meta-

\section{Figure 1. PRISMA chart.}

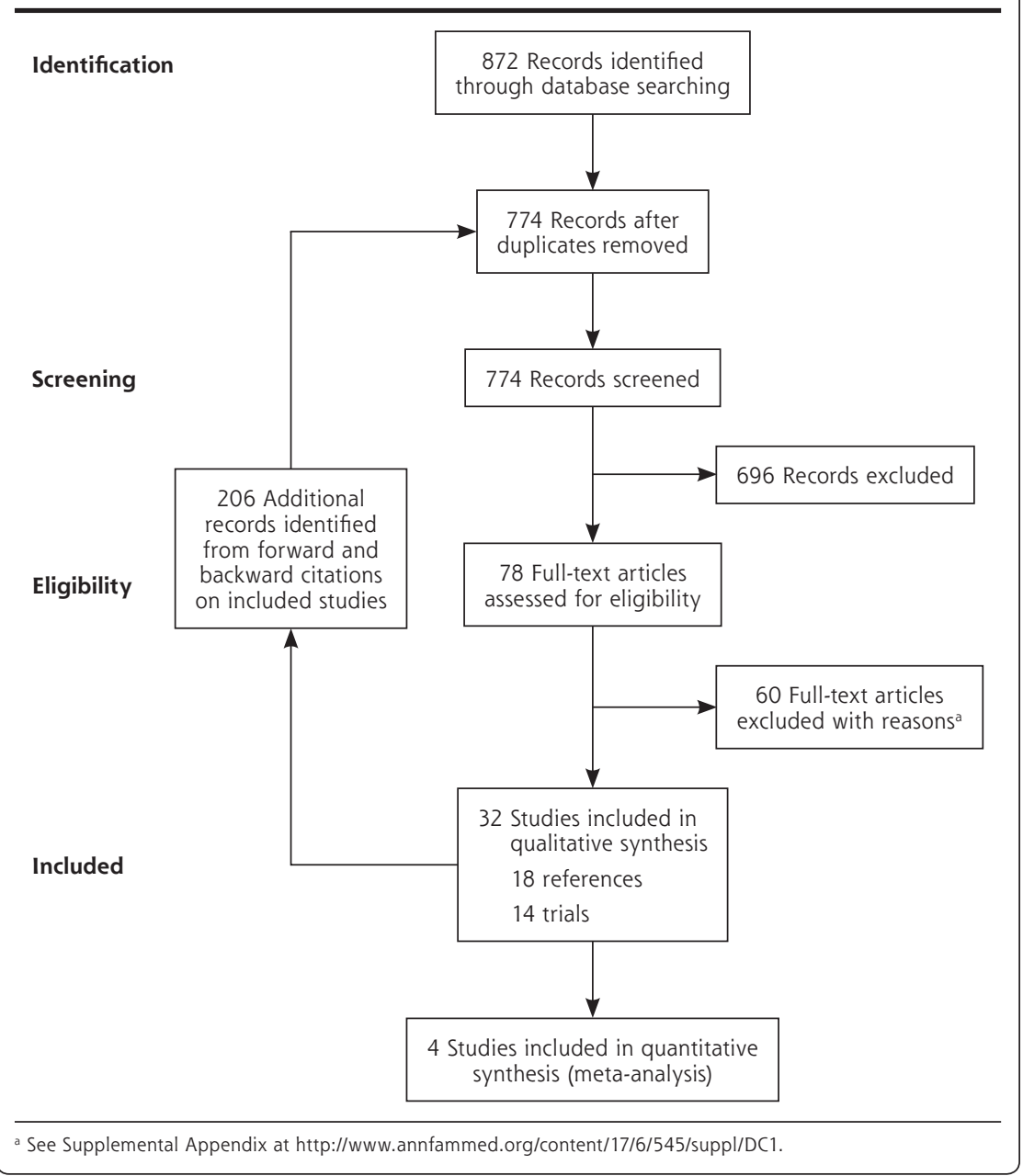

\section{Figure 2. Risk of bias.}

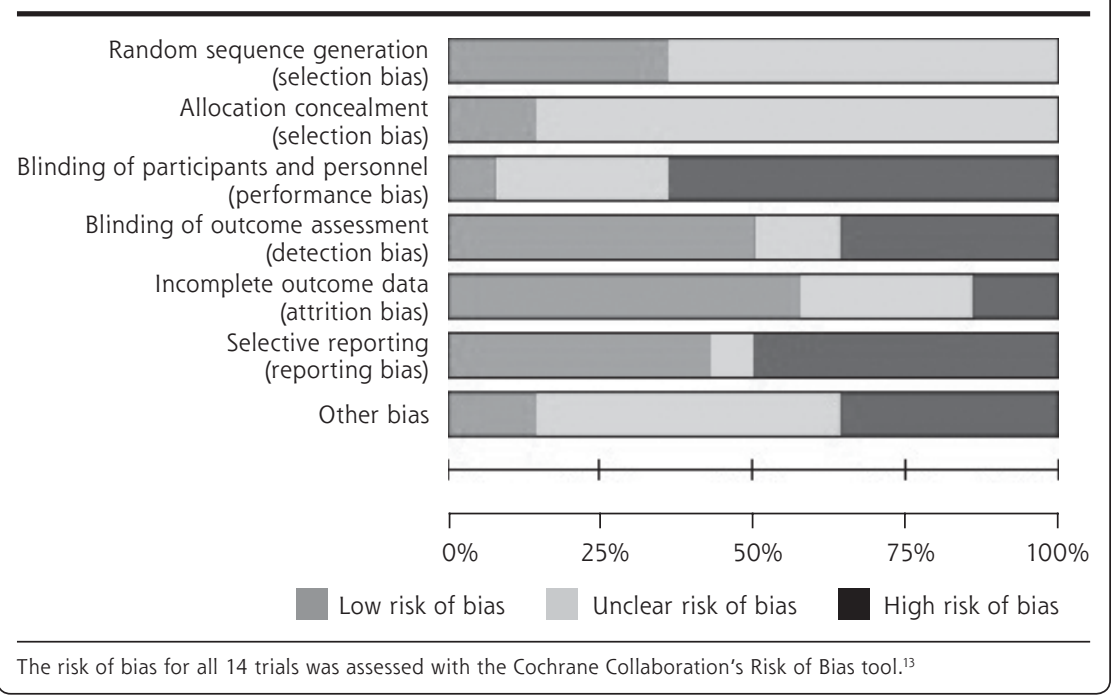

analyzable data, we did not conduct a sensitivity analysis to assess the impact of excluding trials with lower quality (moderate or high risk of bias). Where data were presented as a mean and range, rather than mean and standard deviation, the range was converted to standard deviation. ${ }^{14}$

\section{RESULTS \\ Search Results}

The electronic search identified 872 references, which were supplemented with 206 from forward and backward citations of the included studies (1,078 in total). Removing duplicates left 774. Screening these on title and abstract excluded 696, leaving 78, for which we obtained fulltext articles. Screening these excluded another 60 (Supplemental Table 1 and Supplemental Table 2, available at http://www.AnnFamMed.org/ content/17/6/545/suppl/DC1/), to leave 18 included references that describe 14 trials (Figure 1). No additional publications that met the inclusion criteria were identified from searches of clinical trial registries (Supplemental Table 3, available at http://www.AnnFamMed.org/ content/17/6/545/suppl/DC1/).

\section{Risk of Bias}

The overall risk of bias was high for most trials, particularly for blinding and selective reporting, and uncertain for allocation concealment and random sequence generation (Figure 2).

\section{Characteristics of Included Studies}

All 14 included trials were in English: 9 were full papers, ${ }^{15-23}$ 2 were full papers with conference abstracts, ${ }^{3,24}$ and 3 were conference abstracts alone $e^{25-27}$ (Table 1). 
The trials included 698 participants in total ${ }_{i}$ most trials were small, with only 5 containing more than 50 participants. $8,16,17,21,26$

The unit of randomization was individual patients, except for 3 trials in which hemifaces were randomized $^{16,18,23}$ and 1 trial that randomized by lesion. ${ }^{20}$

Blue light alone was the intervention in 8 trials, ${ }_{1}^{16-20,23}$
2 trials had both a blue-alone arm and a blue/redlight arm, ${ }^{8,26}$ and 4 trials used a blue/red combination light. 3,15,21,27 Three trials combined the light intervention with topical treatments as adjuncts. ${ }^{21,24,25}$

Five trials compared the blue light with no treatment or a sham device. Other comparators included topical cleansers (such as benzoyl peroxide), other

Table 1. Characteristics of Included Studies

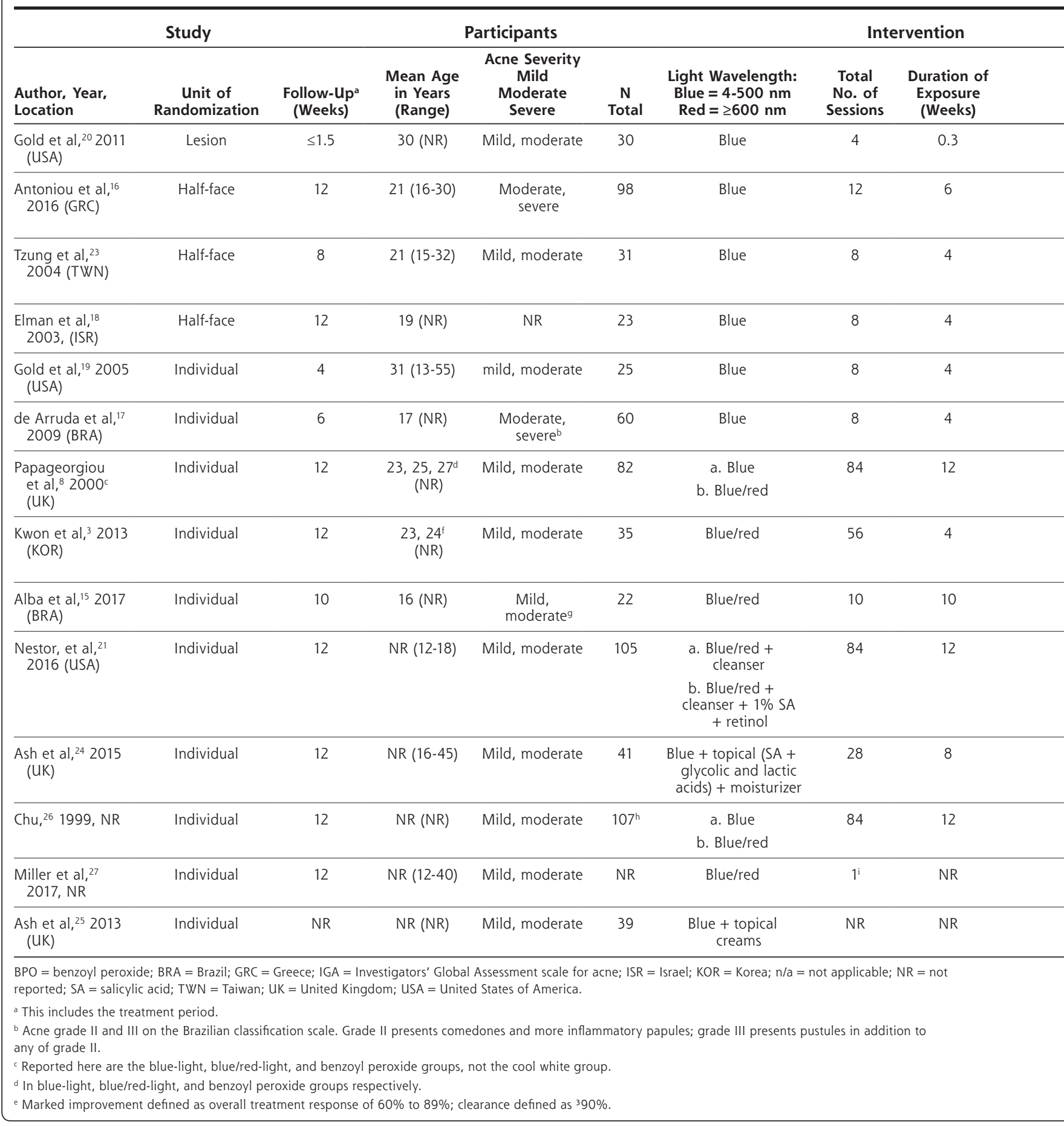


topical agents such as salicylic acid to promote desquamation, or retinol. A few trials had several intervention arms to enable comparison of some of the interventions $^{8,21,26}$ (Supplemental Table 4, available at http:// www.AnnFamMed.org/content/17/6/545/suppl/DC1/).

Follow-up ranged from "up to 10 days" after the first treatment to 12 weeks.
Primary Outcome: Investigator's Assessment of Improvement

Heterogeneity, especially of outcome measures, precluded meta-analyses of the primary outcome (investigator-assessed change), or the secondary outcomes of patient-assessed change, and adverse events (AEs).

Five of the 14 trials reported investigator-assessed improvement quantitatively. Of these, 3 reported significantly greater improvement in acne severity as assessed by investigators for blue or blue/red light, as compared with no treatment or sham device. ${ }^{3,16,23}$ One multiarm trial showed greatest improvement for blue/red light, followed by blue light, and benzoyl peroxide $(P=N R),{ }^{8}$ and another showed greater improvement in blue/red light with or without topicals as compared with benzoyl peroxide $(P=\mathrm{NR}){ }^{21}$

Two trials reported this outcome narratively. One reported that scores were "similar" between blue-light and clindamycin groups, ${ }^{19}$ another reported that more than $80 \%$ of patients in blue/red-light group showed "moderate to marked improvement in their acne," but no results were reported for either the bluelight-only or benzoyl peroxide groups $^{26}$ (Table 1).

\section{Secondary Outcome: Patients' Assessment of Improvement}

Four trials reported the secondary outcome of patient assessment change (2 quantitatively, 2 narratively) and were generally positive.

In 1 trial, the patients were asked to self-assess the severity of their acne at baseline, using the Visual Analogue Scale, with 0 designating disease-free status and 10 designating a state of acne. All patients in both groups (intervention and control) set their baseline status at $10_{i}$, final 
scores in the blue/red group more than halved, to 4.3 , while increasing for the sham group $(P<.05) .{ }^{3}$ In another trial, $75 \%$ of patients in the blue/red group reported clear or marked improvement, compared with $57 \%$ in the blue-light-only and $35 \%$ in the benzoyl peroxide group $(P=\mathrm{NR}){ }^{8}$

Two trials reported this outcome narratively; one reported similar scores between the blue-light and clindamycin groups, ${ }_{19}$ and another reported "moderate to marked improvement" in the blue/red-light group but did not report outcomes for the blue-light-only or benzoyl peroxide group. ${ }^{26}$

\section{Secondary Outcome: Noninflammatory Lesions}

Data were sufficient to pool at weeks 4, 8, and 10-12 for the mean difference between groups in the mean number of noninflammatory lesions treated with blue light or comparator. The difference between groups was not significant at any of the time points or overall (MD 3.47; 95\% CI, -0.76 to $7.71 ; P=0.11$ ) (Figure 3).

\section{Secondary Outcome: Inflammatory Lesions}

Meta-analysis was possible for the mean difference in the mean number of inflammatory lesions at weeks 4,8 , and 10-12. There was no significant difference between patients or lesions treated with blue light or a comparator at any of the time points or overall (MD $0.16 ; 95 \% \mathrm{CI},-0.99$ to $1.31 ; P=0.78$ ) (Figure 4 ).

\section{Secondary Outcome: Adverse Events}

Ten trials reported on AEs (including skin irritation, erythema, dryness, tightness, rashes, peeling, itching, burning, acne flare-ups, changes in pigmentation) in varying degrees of detail.

Figure 3. Mean difference between groups in the mean number of noninflammatory lesions.

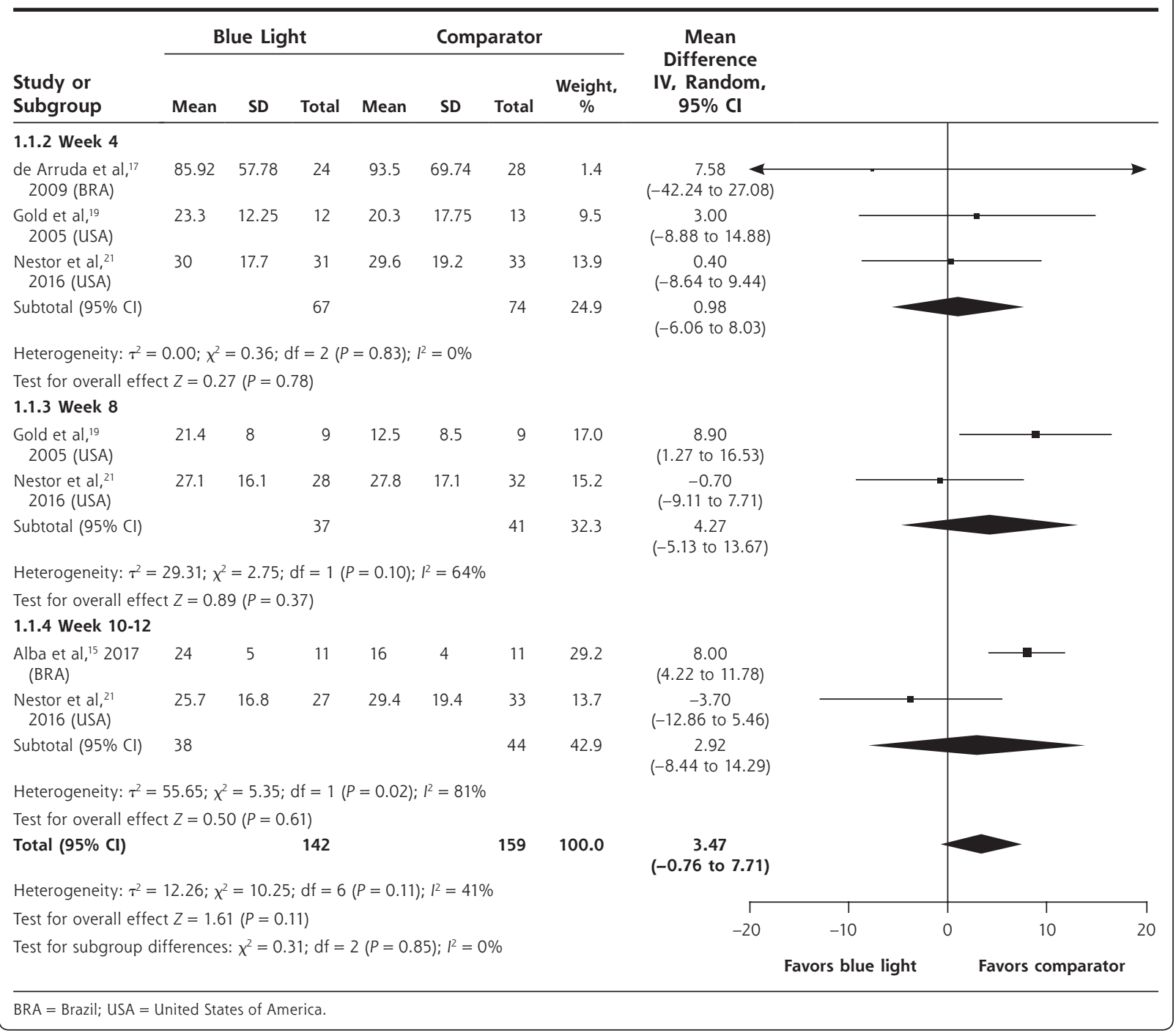


Four trials reported the differences between bluelight and comparator groups in AEs. . $^{817,21,26}$ One trial found no significant differences between groups, except dryness/itch (more frequent with benzoyl peroxide) ${ }_{1}^{8}$ and another found AEs in all treatment groups, but none were more severe than "mild."21 One study reported nearly all patients in benzoyl group reporting AEs vs $23 \%$ in blue light. ${ }^{17}$ One reported $20 \%$ of patients in the benzoyl peroxide group reporting dryness or irritation; percentages in the other groups were unclear. ${ }^{26}$

Two trials reported the number of AEs for the trial overall rather than by treatment group ${ }^{3,16}$; few AEs were reported, and 4 trials reported narratively that the treatment had no side effects or was side-effect free. ${ }^{15,18,24,25}$

\section{DISCUSSION}

This systematic review of blue-light therapy for acne found 14 eligible randomized trials. Methodological and reporting limitations of the trials limit the conclusions that can be drawn about the effect of blue-light therapy. Most trials had small sample sizes and were of limited duration, with none longer than 12 weeks. The risk of detection bias, reporting bias, and performance bias was high in many of the trials. Reporting quality of many trials was also limited, with missing information on interventions and quantitative findings. Only 5 of the included trials quantitatively reported on the primary outcome of investigator-assessed changes in acne severity, and all reported improvements in the intervention group compared with the compara-

Figure 4. Mean difference between groups in the mean number of inflammatory lesions.

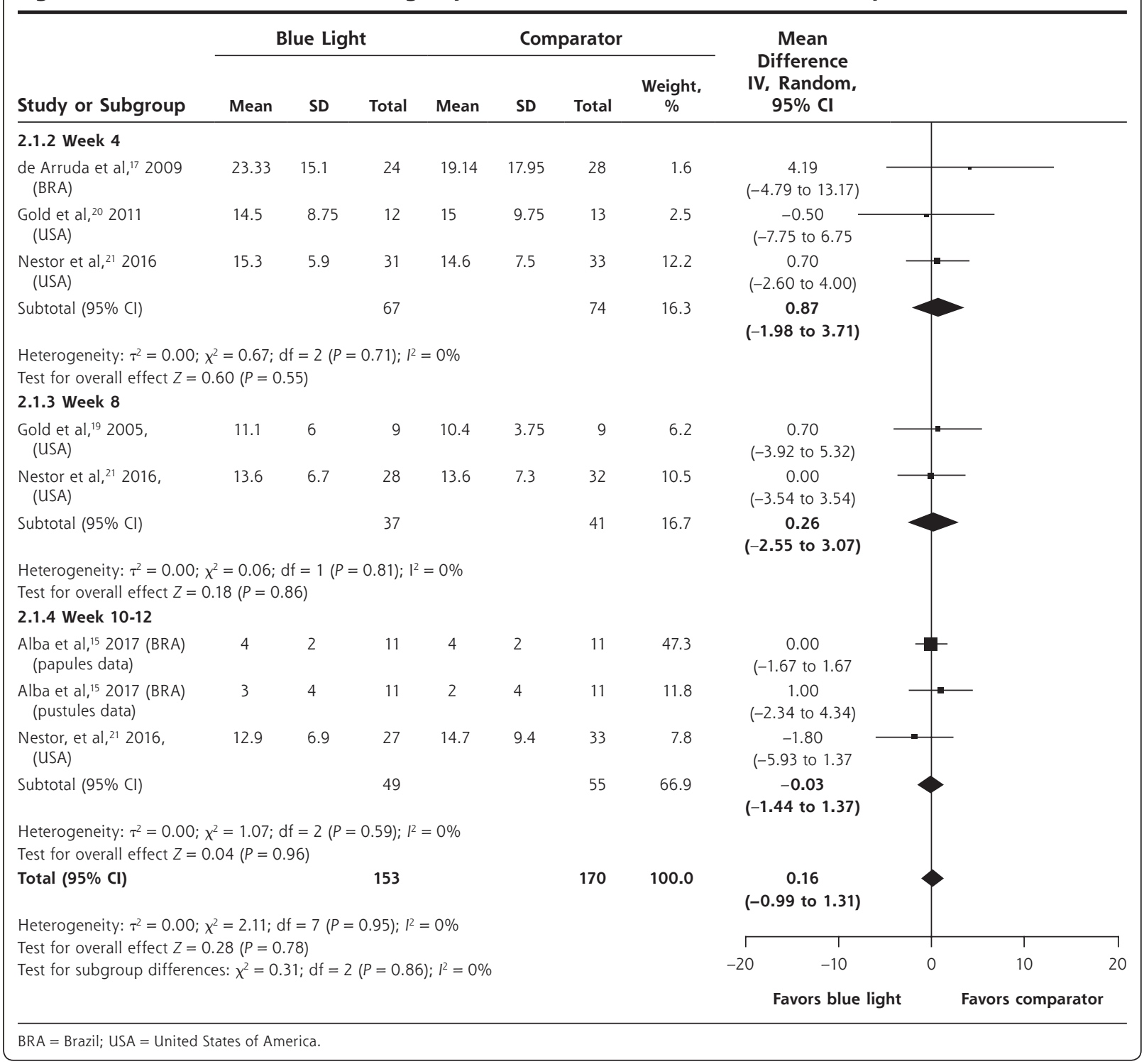


tor, although the proportion of people who reported improvement and the size of improvement varied between studies.

A recent Cochrane review of light therapy for acne ${ }^{11}$ included 71 studies in total and 8 trials that examined the effect of blue light. ${ }^{3,8,17-20,23,24}$ It also pointed out methodological weaknesses in the existing trials, which precluded meta-analysis of the results, and concluded that there is low certainty of the usefulness of blue-light therapy in the treatment of acne. The present systematic review adds 6 trials, 15,16,21,25-27 but persisting limitations in the evidence base for blue-light therapy preclude recommendations about a particular intervention.

Additional randomized controlled trials of blue-light therapy require both more comprehensive reporting and higher methodological rigor. In particular, trials need to enroll a sufficient number of patients to detect clinically important differences, be of sufficiently long duration to be meaningful, and more consistently measure and report outcomes that are important to patients, including AEs. A core outcome set for acne clinical trials has been recently developed and includes the following domains: satisfaction with appearance, long-term acne control, health-related quality of life, extent of scars or marks, signs and symptoms, AEs, and satisfaction with treatment received, work is in progress to identify or develop measures for each of these outcomes. ${ }^{4}$ A more comprehensive reporting of the trials can be facilitated by adherence in reporting of the trials to the Template for Intervention Description and Replication checklist ${ }^{28}$ and the Consolidated Standards of Reporting Trials statement. ${ }^{29}$

Methodological and reporting limitations in currently available trials preclude a firm conclusion about the effectiveness of blue-light therapy for acne. The potential role of blue light in acne is therefore likely to be secondary, following first-line treatments such as topical benzoyl peroxide. In particular, patients and clinicians might consider blue light as an alternative to oral antibiotics, to avoid both AEs and antibiotic resistance. Response should be carefully monitored, however. Patients who are currently considering using blue-light therapy should discuss with their clinicians the possible benefits of the therapy (noting that the estimates of benefit are variable and uncertain, and have not been studied beyond 12 weeks), the potential for AEs (noting that it is difficult to quantify the risk of AEs), and practical details about what the intervention involves and its cost, as well as the benefits, AEs, and practical details of any other treatment options they are considering.

To read or post commentaries in response to this article, see it online at http://www.AnnFamMed.org/content/17/6/545.
Key words: acne vulgaris; humans; light; phototherapy

Submitted November 30, 2018; submitted, revised, March 21, 2019; accepted April 11, 2019.

Funding Support: No funding or other material support was sought or received to perform this work specifically; however, several of the authors (T.H., C.D.M., P.G.) are recipients of funding from the National Health and Medical Research Council Centre for Research Excellence in Minimising Antimicrobial Resistance from Acute Respiratory Infections (CREMARA), and 2 of the authors' salaries (A.M.S., J.C.) are partially funded by CREMARA.

Supplemental materials: Available at http://www.AnnFamMed. org/content/17/6/545/suppl/DC1/.

\section{References}

1. Zaenglein AL, Pathy AL, Schlosser BJ, et al. Guidelines of care for the management of acne vulgaris. J Am Acad Dermatol. 2016;74(5): 945-973.e33.

2. Vos $T$, Flaxman $A D$, Naghavi $M$, et al. Years lived with disability (YLDs) for 1160 sequelae of 289 diseases and injuries 1990-2010: a systematic analysis for the Global Burden of Disease Study 2010. Lancet. 2012;380(9859):2163-2196.

3. Kwon HH, Lee JB, Yoon JY, et al. The clinical and histological effect of home-use, combination blue-red LED phototherapy for mild-tomoderate acne vulgaris in Korean patients: a double-blind, randomized controlled trial. Br J Dermatol. 2013;168(5):1088-1094.

4. Layton AM, Eady EA, Thiboutot DM, Tan J; Acne Core Outcomes Research Network (ACORN) Outcomes Identification Group. Identifying what to measure in acne clinical trials: first steps towards development of a core outcome set. J Invest Dermatol. 2017;137(8): 1784-1786.

5. Dessinioti C, Katsambas A. Propionibacterium acnes and antimicrobial resistance in acne. Clin Dermatol. 2017;35(2):163-167.

6. Walsh TR, Efthimiou J, Dréno B. Systematic review of antibiotic resistance in acne: an increasing topical and oral threat. Lancet Infect Dis. 2016;16(3):e23-e33.

7. Ashkenazi H, Malik Z, Harth Y, Nitzan Y. Eradication of Propionibacterium acnes by its endogenic porphyrins after illumination with high intensity blue light. FEMS Immunol Med Microbiol. 2003;35(1): 17-24.

8. Papageorgiou P, Katsambas A, Chu A. Phototherapy with blue (415 $\mathrm{nm})$ and red $(660 \mathrm{~nm})$ light in the treatment of acne vulgaris. $\mathrm{Br}$ J Dermatol. 2000;142(5):973-978.

9. Thiboutot D, Gollnick H, Bettoli V, et al; Global Alliance to Improve Outcomes in Acne. New insights into the management of acne: an update from the Global Alliance to Improve Outcomes in Acne group. J Am Acad Dermatol. 2009;60(5)(suppl):S1-S50.

10. Seth D, Wang S, Ezaldein HH, Merati M, Scott JF. Over-the-counter light therapy for acne: a cross-sectional retrospective analysis. Dermatol Online J. 2019;25(1):13030/qt8sq5j6vk.

11. Barbaric J, Abbott R, Posadzki P, et al. Light therapies for acne. Cochrane Database Syst Rev. 2016;9:CD007917.

12. Simon C, Glasziou P. A truly "HANDI" resource. Br J Gen Pract. 2019; 69(679):80-81.

13. Higgins J, Green S, eds. Cochrane Handbook for Systematic Reviews of Interventions. Version 5.1.0. London: The Cochrane Collaboration. Updated Mar 2011.

14. Hozo SP, Djulbegovic B, Hozo I. Estimating the mean and variance from the median, range, and the size of a sample. BMC Med Res Methodol. 2005;5(1):13. 
15. Alba MN, Gerenutti M, Yoshida VM, Grotto D. Clinical comparison of salicylic acid peel and LED-laser phototherapy for the treatment of acne vulgaris in teenagers. J Cosmet Laser Ther. 2017;19(1):49-53.

16. Antoniou C, Dessinioti C, Sotiriadis D, et al. A multicenter, randomized, split-face clinical trial evaluating the efficacy and safety of chromophore gel-assisted blue light phototherapy for the treatment of acne. Int J Dermatol. 2016;55(12):1321-1328.

17. de Arruda LH, Kodani V, Bastos Filho A, Mazzaro CB. [A prospective, randomized, open and comparative study to evaluate the safety and efficacy of blue light treatment versus a topical benzoyl peroxide $5 \%$ formulation in patients with acne grade II and III]. An Bras Dermatol. 2009;84(5):463-468.

18. Elman $M$, Slatkine $M$, Harth $Y$. The effective treatment of acne vulgaris by a high-intensity, narrow band 405-420 nm light source. J Cosmet Laser Ther. 2003;5(2):111-117.

19. Gold MH, Rao J, Goldman MP, et al. A multicenter clinical evaluation of the treatment of mild to moderate inflammatory acne vulgaris of the face with visible blue light in comparison to topical $1 \%$ clindamycin antibiotic solution. J Drugs Dermatol. 2005;4(1):64-70.

20. Gold MH, Sensing W, Biron JA. Clinical efficacy of home-use bluelight therapy for mild-to moderate acne. J Cosmet Laser Ther. 2011; 13(6):308-314.

21. Nestor MS, Swenson N, Macri A, Manway M, Paparone P. Efficacy and tolerability of a combined $445 \mathrm{~nm}$ and $630 \mathrm{~nm}$ over-the-counter light therapy mask with and without topical salicylic acid versus topical benzoyl peroxide for the treatment of mild-to-moderate acne vulgaris. J Clin Aesthet Dermatol. 2016;9(3):25-35.

22. Sakamoto FH, Izikson L, Lewis W, et al. Preliminary results of a pilot prospective ramdomized double-blinded control study to compare a new inhibitory PDT method to conventional ALA-PDT for the treatment of recalcitrant moderate-severe acne. Lasers Surg Med. $2012 ; 44: 8$.
23. Tzung TY, Wu KH, Huang ML. Blue light phototherapy in the treatment of acne. Photodermatol Photoimmunol Photomed. 2004;20(5): 266-269.

24. Ash C, Harrison A, Drew S, Whittall R. A randomized controlled study for the treatment of acne vulgaris using high-intensity 414 nm solid state diode arrays. J Cosmet Laser Ther. 2015;17(4):170-176.

25. Ash C, Drew S, Harrison A, Grant T, Mills D, Herbert K. Home use efficacy and safety study for mild to moderate acne using high intensity $414 \mathrm{~nm}$ solid state diode arrays. Lasers Med Sci. 2013; 28(5):1216.

26. Chu A. Phototherapy with blue (415) and red (660) light in the treatment of mild to moderate acne vulgaris. J Eur Acad Dermatol Venereol. 1999;12(suppl 2):S99.

27. Miller D, Friscia D, Fitzgerald L, Wu MM. Clinical efficacy and tolerability of new low-level blue and red light therapy technology in the treatment of mild to moderate acne. J Am Acad Dermatol. 2017; 76(6):AB73.

28. Hoffmann TC, Glasziou PP, Boutron I, et al. Better reporting of interventions: Template for Intervention Description and Replication (TIDieR) checklist and guide. BMJ. 2014;348:g1687.

29. Schulz KF, Altman DG, Moher D; CONSORT Group. CONSORT 2010 statement: updated guidelines for reporting parallel group randomised trials. BMJ. 2010;340:c332. 